\title{
FILTERED POLYNOMIAL APPROXIMATION ON THE SPHERE
}

\author{
YUGUANG WANG
}

(Received 31 August 2015; first published online 27 October 2015)

\begin{abstract}
2010 Mathematics subject classification: primary 42C40; secondary 33C55, 65D32, 43A90, 33 C45. Keywords and phrases: polynomials, sphere, filter, localisation, needlets, quadrature rules.
\end{abstract}

Localised polynomial approximations on the sphere have a variety of applications in areas such as signal processing, geomathematics and cosmology. Filtering is a simple and effective way of constructing a localised polynomial approximation. In this thesis, we investigate the localisation properties of filtered polynomial approximations on the sphere. Using filtered polynomial kernels and a special numerical integration (quadrature) rule we construct a fully discrete needlet approximation.

The localisation of the filtered approximation can be seen from the localisation properties of its convolution kernel. As a particular example, we investigate the localisation of the filtered Jacobi kernel, which includes the convolution kernel, for filtered approximation on the sphere. We prove the precise relationship between the filter smoothness and the decay rate of the corresponding filtered Jacobi kernel over local and global regions.

The difference in localisation properties between Fourier and filtered approximations can be illustrated by their Riemann localisation. We show that the Riemann localisation property holds for the Fourier-Laplace partial sum for sufficiently smooth functions on the two-dimensional sphere, but does not hold for spheres of higher dimension. We then prove that the filtered approximation with sufficiently smooth filter has the Riemann localisation property for spheres of any dimension.

Filtered convolution kernels with a special filter become spherical needlets, which are highly localised zonal polynomials on the sphere with centres at the nodes of a suitable quadrature rule. The original semidiscrete spherical needlet approximation $[1,2]$ has coefficients defined by inner product integrals. We use an appropriate quadrature rule to construct a fully discrete version. We prove that the fully discrete spherical needlet approximation is equivalent to filtered hyperinterpolation, that is,

Thesis submitted to the University of New South Wales in March 2015; degree approved on 27 May 2015; supervisors Ian H. Sloan, Robert S. Womersley and Michael G. Cowling.

(c) 2015 Australian Mathematical Publishing Association Inc. 0004-9727/2015 \$16.00 
to a filtered Fourier-Laplace partial sum with inner products replaced by appropriate quadrature sums. From this, we establish error bounds for the fully discrete needlet approximation of functions in Sobolev spaces on the sphere. The power of the needlet approximation for local approximation is shown by numerical experiments that use low-level needlets globally, together with high-level needlets in a local region.

\section{References}

[1] F. Narcowich, P. Petrushev and J. Ward, 'Decomposition of Besov and Triebel-Lizorkin spaces on the sphere', J. Funct. Anal. 238(2) (2006), 530-564.

[2] F. J. Narcowich, P. Petrushev and J. D. Ward, 'Localized tight frames on spheres', SIAM J. Math. Anal. 38(2) (2006), 574-594.

YUGUANG WANG, School of Mathematics and Statistics,

UNSW Australia, Sydney, NSW 2052, Australia

e-mail: yuguang.e.wang@gmail.com 